\title{
High speed phase modulators for silicon photonic integrated circuits: a role for lithium niobate?
}

\author{
Roel Baets ${ }^{a, b, *}$ and Bart Kuyken ${ }^{a, b}$ \\ ${ }^{a}$ Ghent University-imec, Photonics Research Group, Gent, Belgium \\ ${ }^{b}$ Ghent University, Center for Nano- and Biophotonics, Gent, Belgium
}

Lithium niobate (LN) has been and still is a prominent material for high speed optical modulators. The performance of such LN-modulatorsas a stand-alone building block for optical systems-has been unchallenged for many years. But in many ways it has proven to be very hard to integrate lithium niobate modulators with other functions on a single optical chip. As a result, scientific interest in this material has been fading away. As a consequence of the success in the development of thin film lithium niobate, recent reports ${ }^{1,2}$ are starting to change this and may open a route to integrated photonic chips including LN-modulators. Furthermore these integrated LN-modulators take advantage of recent concepts (e.g., tight confinement through a high refractive indexcontrast) and integration technologies (e.g., die-to-wafer bonding), allowing them to outperform their more conventional predecessors in sheer performance. So we can rightfully ask the question: is lithium niobate back in business? This paper puts this question in perspective.

Ever since the seminal paper by Stewart Miller in $1969^{3}$ there has been the ambition to build integrated optical systems on a chip, very much following the technical and economic logic behind the success of electronic integrated circuits. In such integrated optical circuits-now more commonly called photonic integrated circuits or PICs-a variety of optical building blocks would be integrated on a planar substrate and would be interconnected by means of optical waveguides. Actually the building blocks themselves were typically also waveguide-based and would therefore take advantage of optical field confinement in compact cross-sections over distances many orders of magnitude beyond the Rayleigh-range of optical beams diffracting in free space.

For several decades most of the research efforts would focus on the key building blocks: passive optical functions such as spectral filtering, electro-optical modulation, optical amplification, laser emission, light detection, and more. Given the difficulty of achieving all these functions in a single material system, the field diverged. For purely passive functions low loss dielectric materials were chosen, with silica being an important example. The term "planar lightwave circuit" (PLC) was typically used for such passive chips. For light amplification and laser emission III-V semiconductors were the primary material of choice, with the exact material choice dictated by the wavelength of operation. Here one would take advantage of the power of epitaxy to create layer structures that would at the same time provide confinement for free charge carriers (electrons and holes) as well as for photons. For optical phase modulation one would resort to electro-optic materials with a strong Pockels effect. The Pockels effect is an ultra-fast and optically broadband electro-optic effect in which an electric field induces changes to the relative position of bound electrons and atomic nuclei, which translates into a refractive index change that scales linearly with the applied electric field. This allows for pure phase modulators. The Pockels effect can only occur in materials that lack inversion symmetry, such as in non-centro-symmetric crystals (like LN) or poled polymers.

*Address all correspondence to Roel Baets, E-mail: roel.baets@ugent.be

(C) The Authors. Published by SPIE and CLP under a Creative Commons Attribution 4.0 Unported License. Distribution or reproduction of this work in whole or in part requires full attribution of the original publication, including its DOI. [DOI: 10.1117/1 .AP.1.3.030502]
$\mathrm{LN}$ has been the most successful material for high speed optical modulation, due to its strong Pockels effect, first as a bulk material and later in the form of planar waveguide-based components. While the basic modulation effect is based on index modulation, and therefore optical phase modulation, it is relatively straightforward to convert that into amplitude modulation, either by combining the modulator with polarizers or by making use of an interferometric construct in which one path is phase modulated and another path is not or inversely modulated. Upon recombination of both paths amplitude modulation results. The key performance metric of such a modulator is the voltage $V_{\pi}$ needed for a $\pi$ phase shift of the optical beam (translating into onoff amplitude modulation). This voltage can be scaled down by making the device length $L$ larger. Actually, the key metric is the $V_{\pi} L_{\pi}$ product, expressed in $\mathrm{V} \cdot \mathrm{cm}$. Typically $\mathrm{LN}$-modulators have $V_{\pi} L_{\pi}$-product of the order of $10-20 \mathrm{~V} \cdot \mathrm{cm}$. But making the device longer creates other problems. Not only is compactness an asset in its own right, but furthermore the waveguide losses per unit length $\alpha$ (in $\mathrm{dB} / \mathrm{cm}$ ) yield a maximum meaningful length for the device. In this context the product $V_{\pi} L \alpha$ (expressed in $\mathrm{V} \cdot \mathrm{dB}$ ) is also often used as a figure of merit for modulators. Another length-dependent property relates to the bandwidth of the electro-optic conversion process. The intrinsic bandwidth of the Pockels effect is ultra-high but the practical bandwidth is generally limited by resistor-capacitor effects of the electrical circuitry, which scale with the length. Moreover, for long devices, the modulator cannot be seen as a lumped electrical element and a mismatch between the spatial distribution of the modulated optical field and the applied electric field can occur as a result of the difference between the group velocities of the optical and electric wave respectively. In this context the highest bandwidth designs almost invariably use a traveling wave approach for the electric drive signal, such that it has the same propagation speed as the optical wave. With this approach modulation bandwidths up to about $40 \mathrm{GHz}$ have been reported.

The status quo as described above has been broken recently through the use of high-index-contrast photonics. Indeed, the waveguides described earlier were typically low-index-contrast waveguides in which in-diffused ions provided the (very weak) index contrast between core and cladding, with a weak optical confinement as a result (Fig. 1). In Ref. 2 a drastically different approach is proposed and demonstrated. The LN-waveguide is a thin and narrow strip of LN embedded between silica claddings. This high-index contrast waveguide ensures a very compact guided mode cross-section, as a result of which the electrodes can be positioned much closer to the waveguide. As a result, for the same applied voltage a much stronger field can be induced without inducing a high optical loss. Additionally the design allows for independently tuning the speed of the light and the electrical travelling wave without sacrificing the overlap between the electric field and the optical field. The high mismatch between the dielectric constants at optical and microwave frequencies is tackled in a traditional LN modulator by introducing a silica dielectric layer between the $\mathrm{LN}$ and the electrodes. This however reduces the overlap. In Ref. 2 the velocity of the microwave is adjusted by tuning the buried oxide layer's thickness which has very little influence on the optical mode. The resulting device boosts an impressive performance with a $V_{\pi} L_{\pi}$-product between 2 and $3 \mathrm{~V} \cdot \mathrm{cm}$, 
(a)

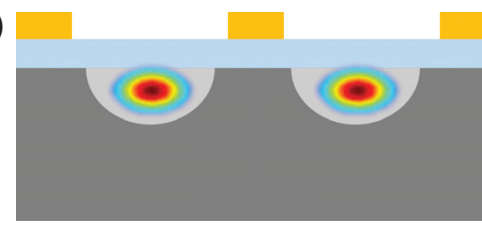

Metal contact (b)

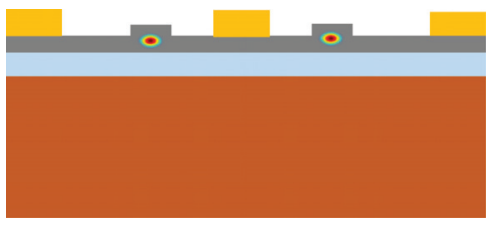

(c)

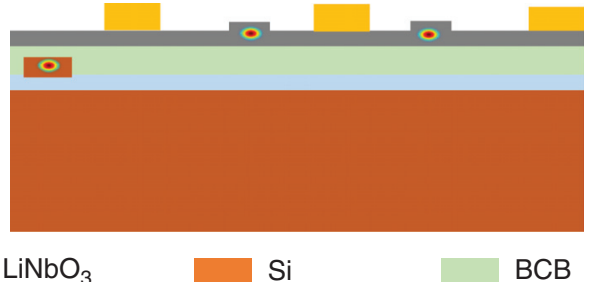

Fig. 1 (a) A traditional lithium niobate modulator; (b) a cross-section of the modulator of Ref. 2; (c) a cross-section of the modulator of Ref. 1.

a modulation bandwidth up to $100 \mathrm{GHz}$ and a device loss well below $0.5 \mathrm{~dB}$. While the used approach may seem relatively straightforward, it has taken non-trivial technological advances to make it happen. The crystalline thin film of $\mathrm{LN}$ is manufacturing by means of wafer bonding to form a so-called LN-on-insulator wafer (NANOLN). The etching of ridge waveguides is notably difficult in the case of LN and takes a carefully optimized reactive ion etching process in order to yield low-loss waveguides.

However impressive the performance of this result is, the LN-modulator is still a discrete component. In the past few decades the field of photonic integration has been moving in the direction of platforms that enable the integration of photonic circuits with a diversity of passive and active components within one circuit. This is a non-trivial task because different materials are good at different functionalities. Therefore integrating multiple functionalities within one materials platform typically leads to compromises on performance, at least in an early phase. Nevertheless the benefits in terms of cost and reliability resulting from the integration on one platform are important enough to make it highly relevant from an industrial point of view. Moreover, when many research and industrial actors focus their efforts on one platform, a virtuous circle arises in which advancement of performance accelerates and economy-of-scale effects create an ecosystem in which fabs and fabless users can thrive.

While the notion of a one-platform-approach has been tried with multiple materials, it is quite clear by now that silicon photonics is gaining the largest momentum. Silicon photonics makes use of the tool sets and process flows found in CMOS fabs to manufacture photonic integrated circuits with silicon of silicon-nitride-based waveguides, interconnecting a diversity of components. The most common modality is to use silicon-on-insulator (SOI) wafers in which passive silicon waveguides are formed along with waveguides with $\mathrm{p}-\mathrm{n}$ junctions to form phase modulators, based on the modulation of the free carrier concentration, as well as monolithic germanium detectors. With this combination a variety of impressive products has been developed, in particular high symbol rate (at least up to 40 Gbaud) optical transceivers for the telecom and datacom market.

Silicon and germanium do lack the proper material characteristics however for all functionalities that one may wish for. Not only is light emission highly inefficient, also the Pockels effect is absent. Therefore it is not possible to build a pure phase modulator in a similar way as is the case in LN. While the free-carrier-based modulators mentioned earlier perform quite well, they have intrinsic limitations in speed and optical loss. Moreover, they do not allow for pure phase modulation because there is residual amplitude modulation due to free carrier induced loss. Already today they are used more or less at a performance level close to physical limits, but the market is calling for even higher performance so as to address the insatiable demands for bandwidth. This is where heterogeneous integration comes in, whereby a diversity of materials is co-integrated on a silicon photonics platform. In the case of amorphous materials the co-integration can be done by straightforward thin film deposition (sputtering, plasma-enhanced deposition, atomic layer deposition, etc.), but in the case of crystalline materials one needs to resort to more sophisticated methods. One may try to use an epitaxial growth technique directly on silicon (or a deposition technique followed by anneal to create a crystalline phase), but this is typically very demanding and only possible for certain material combinations. Invariably it also involves a process step at elevated temperatures. The alternative is to start from a crystalline thin film grown onto a different host substrate and then somehow transfer and bond that film to the silicon photonics wafer. Methods to do so have become relatively mature in recent years. Either they use bonding of the thin film to the silicon wafer with subsequent removal of the host substrate ${ }^{4}$ or they use lift-off of the thin film (and possibly even structured devices) from the host substrate followed by bonding to the silicon wafer. The latter technique is known as micro-transfer printing. ${ }^{5}$ These techniques have led to many impressive demonstrations of co-integration of III-V devicesin particular laser diodes and semiconductor optical amplifiers-with silicon PICs. But also a variety of other crystalline materials have been co-integrated with silicon PICs.

In Ref. 1 the co-integration of LN-modulators with silicon waveguides is done with the help of die-to-wafer bonding. Although such an approach has been demonstrated before, ${ }^{6}$ the performance here is boosted. Instead of relying on hybrid modes where part of the light is in the silicon and part of the light is confined in the lithium niobate layer, the authors make sure all of the light is in the lithium niobate layer. They achieve this with vertical adiabatic couplers. These consist of a silicon inverted taper and a superimposed straight, $\mathrm{LN}$ waveguide. Due to the high confinement in the $\mathrm{LN}$ waveguides they are able to make efficient $\mathrm{LN}$ modulators with a $V_{\pi} L_{\pi}=2-2.5 \mathrm{~V} \cdot \mathrm{cm}$ while the routing was done in the silicon waveguide layer. Also in this study a travelling wave design was achieved. This allowed for an electro-optic bandwidth of $70 \mathrm{GHz}$.

These results show the potential of how miniaturization enabled by high index contrast can enhance the performance of devices. By using the high confinement in thin film layers very efficient modulators result. Moreover, by relying on a heterogeneous integration technique, Ref. 1 shows the integration of $\mathrm{LN}$ on a silicon photonics platform. These results pave the way to modulators that can operate at very low voltages at high speeds on silicon. However, while LN is maybe the best known electro-optic material, it is certainly not the only material that can be integrated on a silicon photonics platform. Other research teams have demonstrated high speed modulators that are based on highly electro-optic polymers, ${ }^{7}$ layers of barium titanium oxide $(\mathrm{BTO})^{8}$ or lead zirconate titanate (PZT). ${ }^{9}$ These materials have even a stronger electro-optic effect as compared to LN. The future will tell which of these materials will end up in the new generation of high speed transceivers as well as in other applications that require low loss phase modulation. 


\section{References}

1. M. He et al., "High-performance hybrid silicon and lithium niobate Mach-Zehnder modulators for 100 Gbit s $^{-1}$ and beyond," Nat. Photonics 13(5), 359-364 (2019).

2. C. Wang et al., "Integrated lithium niobate electro-optic modulators operating at CMOS-compatible voltages," Nature 562(7725), 101-104 (2018).

3. S. E. Miller, "Integrated optics: an introduction," Bell Syst. Tech. J. 48(7), 2059-2069 (1969).

4. S. Keyvaninia et al., "Ultra-thin DVS-BCB adhesive bonding of III-V wafers, dies and multiple dies to a patterned silicon-oninsulator substrate," Opt. Mater. Express 3(1), 35-46 (2013).

5. A. De Groote et al., "Transfer-printing-based integration of singlemode waveguide-coupled III-V-on-silicon broadband light emitters," Opt. Express 24(13), 13754-13762 (2016).

6. A. J. Mercante et al., "Thin film lithium niobate electro-optic modulator with terahertz operating bandwidth," Opt. Express 26(11), 14810-14816 (2018).

7. L. Alloatti et al., "100 GHz silicon-organic hybrid modulator," Light Sci. Appl. 3(5), e173 (2014).

8. S. Abel et al., "Large Pockels effect in micro-and nanostructured barium titanate integrated on silicon," Nat. Mater. 18(1), 42-47 (2019).

9. K. Alexander et al., "Nanophotonic Pockels modulators on a silicon nitride platform," Nat. Commun. 9(1), 3444 (2018).

Roel Baets is a full professor at Ghent University and leads the Photonics Research Group of Ghent University, a research group associated with IMEC. He has made a broad variety of contributions to the field of photonic integrated circuits, both in silicon and in III-V semiconductors. His current research interests focus on photonic integrated components for new application areas, such as smart sensors and biomedical instrumentation. He has been granted several scientific prizes and is a fellow of IEEE, EOS, and OSA. He is the director of the Center for Nano- and Biophotonics at UGent. He has been awarded an ERC Advanced Grant and has received a Methusalem Grant from the Flemish government. He coordinates ePIXfab, the European Alliance for Silicon Photonics.

Bart Kuyken received the BS and MS degrees in electrical engineering and the BS degree in applied physics from Ghent University in 2008. He received the MS degree in electrical engineering from Stanford University in 2009. In 2013, he received the PhD degree from Ghent University for the thesis "Four-wave-mixing in dispersion-engineered silicon nanophotonic circuits for telecommunication and sensing applications." As a postdoc, he worked in the field of nonlinear optics. He was a visiting scientist at the IBM TJ Watson Research Center (U.S.) in 2011 and 2012, and at the Max Planck Institute for Quantum Optics (D) in 2013 and 2015. His work includes the integration of nonlinear optical functions in silicon photonics waveguides, 2-D materials, and $\mathrm{THz}$ waveguide integration. In 2015, he was appointed an assistant professor in the Faculty of Engineering of Ghent University. He has been granted an ERC Starting Grant for the development of on-chip frequency combs. 